\title{
Deserción estudiantil en posgrados semipresenciales de la Universidad Estatal a Distancia (UNED), Costa Rica: ¿Deserción o retraso?
}

\author{
Zaidett Barrientos y Roy Umaña \\ Universidad Estatal a Distancia, 474 - 2050 San Pedro Montes de Oca, San José, Costa Rica; zbarrientos@uned.ac.cr; rumana@uned.ac.cr
}

Recibido 6-VIII-2009 Corregido 13-IX-2009 Aceptado 21-IX-2009

\begin{abstract}
Student attrition in posgraduate blended programs at the Universidad Estatal a Distancia (UNED), Costa Rica: Desertion or delay? Postgraduate student attrition in blended (traditional and distance) education programs has been little studied. Several theoretical categories have been developed to justify attrition: psychological, academic, sociological, economic, organizational, supply and demand of higher education and the market, and interaction. Through surveys, interviews and institutional data the case of a blended learning academic master program is analyzed. It was found that the problem must be rethought as a delay in graduation (68\%). Desertion calculated according to academic regulations is $87 \%$, but according to the intention expressed by the students is $19 \%$, of which $6 \%$ transfer to other institutions. Besides this, it takes on average 16 months to submit the candidacy exam although by regulations it is expected to do so in 6 months. Therefore, applying appropriate strategies may lower the dropout rate to about 15\%. Desertion (as intention expressed by the students) is negatively correlated with their opinion about: 1) degree of concordance between the career's output profile and the labor market needs, 2) teachers teaching skills and 3) relationship between the work performed by the student and the master programm. None of the existing theoretical categories fully explain the results, so it is considered that the determining factors in the dropout may vary by sociocultural and institutional situation of each case. To reduce dropout improvements must be made in: 1) teaching skills, 2) curriculum, 3) students selection, 4) subject's contents of the first block, 5) admission of undergraduate students from the same institution and 7) induction to a blended learning system. To reduce delays in graduation people in charge must: 1) restructure the seminary courses, 2) strengthen research in the courses, 3) give more supervision and assistance to student's research project, 4) fit regulation times to students that work full time and 5) select students who really want an academic program (not a professional program).
\end{abstract}

\section{KEY WORDS}

Postgraduate student attrition, delayed graduation, student retention, higher education dropouts.

\section{RESUMEN}

La deserción estudiantil para posgrados semipresenciales ha sido poco estudiada. Se han desarrollado varias categorías teóricas para justificar la deserción: psicológica, académica personal, sociológica, económica, organizacional, oferta y demanda de la educación superior y del mercado, e interacción. Mediante encuestas, entrevistas y datos institucionales se analiza el caso de una maestría académica semipresencial. Se encontró que el problema debe repensarse como un retraso en la graduación (68\%), pues la deserción calculada según el reglamento académico es del $87 \%$, pero según la intención expresada por los estudiantes es de $19 \%$, de los cuales el $6 \%$ corresponde a traslados a otras instituciones, además, en promedio tardan 16 meses en presentar el examen de candidatura cuando reglamentariamente se espera que lo hagan en 6 meses. Por lo tanto, aplicando las estrategias adecuadas es posible bajar la tasa de deserción a alrededor del 15\%. La deserción (según intención de los estudiantes) se relaciona negativamente con la opinión que tengan de: 1) grado de concordancia entre el perfil de salida profesional y las necesidades del mercado laboral, 2) capacidad docente de los profesores y 3) nivel de relación entre el trabajo que desempeña el estudiante y la maestría. Ninguna de las categorías teóricas existentes explica plenamente los resultados, por lo que se considera que los factores determinantes en la deserción pueden variar según la situación sociocultural e institucional de cada caso. Para disminuir la deserción se recomienda mejorar: 1) la capacidad docente, 2) el diseño curricular, 3) la selección de estudiantes, 4) el contenido del primer bloque de materias, 5) el ingreso de estudiantes de grado de la misma institución y 7) la inducción a un sistema semipresencial. Para disminuir el retraso en la graduación se recomienda: 1) reestructurar los cursos de seminario de tesis, 2) fortalecer la investigación en los cursos, 3) dar mas acompañamiento en el proyecto de investigación, 4) ajustar los tiempos reglamentarios y 5) seleccionar estudiantes que quieren una maestría académica.

\section{PALABRAS CLAVE}

Abandono estudiantil, retraso en graduación, postgrados universitarios, permanencia estudiantil. 
El estudio del abandono se ha llevado a cabo en diferentes partes del mundo pero se ha hecho de forma asincrónica y con enfoques distintos. La deserción de los estudios a nivel preuniversitario ha sido ampliamente estudiado desde la década de 1960 (Donoso \& Schiefelbein 2007), también ha sido reconocido como un problema común en todas las universidades a nivel de grado universitario. En Estados Unidos el tema fue abordado principalmente entre las décadas de 1960 a 1990 con modelos teóricos que luego fueron probados con casos reales. Se usó análisis de regresión para determinar los factores determinantes en la deserción de los estudios (Bean 1980, Allen 1999, Murtaugh et al. 1999, Ishitani 2003). En cambio en el ámbito latinoamericano generalmente el tema se discutió en términos de su magnitud (Himmel 2002), y los estudios sobre las razones por las que se presenta empezaron a ser publicados formalmente hasta en la década del 2000 ( $\mathrm{Hi}$ mmel 2002, Donoso \& Schiefelbein 2007, Montero et al. 2007). En el caso de Costa Rica el tema se ha estudiado desde la década de 1980 (Monge 1981, Lobo 1998, Brenes 2000, Umaña 2004, entre otros), pero son muy pocas las publicaciones formales (Méndez 1997, Segura \& Chacón 1997, Montero et al. 2007).

Fue a fines de la década de 1990 que la deserción estudiantil a nivel de posgrados universitarios atrajo la atención de algunos investigadores (Segura \& Chacón 1997, Chyung et al. 1998, Lovitts \& Nelson 2000, Dubs 2005, Park 2005). No obstante, todavía son muy escasos los estudios formales y no se conocen bien las razones que inducen la deserción en este nivel en las distintas sociedades. Esto ocurre a pesar de que la globalización de medios tecnológicos ha permitido que ese proceso de análisis, a diferencia de lo que sucedió con el del abandono estudiantil a nivel de grado, se de casi simultáneamente en varias regiones (Lovitts 2001, Park 2005, Donoso \& Schiefelbein 2007).

El análisis de la deserción estudiantil en los posgrados universitarios es importante pues los procesos de acreditación de los programas consideran a los índices de abandono como indicadores institucionales (García 1987, Latiesa 1992, Dubs 2005). Además, la deserción es preocupante por estar asociada con el fracaso personal (Dubs 2005) y lleva a cuantiosos gastos económicos ( $\mathrm{Hi}$ mmel 2002, Dubs 2005, Rivera et al. 2005,) del estudiante y de las instituciones (Lovitts \& Nelson 2000), sobre todo si se desea acreditar el programa. El estudio de este tema también es importante ya que la sociedad espera que en los estudios de posgrado se genere, por medio de investigaciones, una gran cantidad de información nueva de alta calidad $y$, además, promueva el pensamiento crítico y el involucramiento con las comunidades (UNESCO 2009), por lo que el avance de la sociedad se ve entorpecido si los estudiantes abandonan los estudios a este nivel (Dubs 2005, Lovitts 2005).

Aunque los enfoques teóricos sobre la deserción han sido creados en un contexto de pregrado y grado, también pueden ser aplicados a posgrados universitarios (Himmel 2002). Los modelos teóricos y enfoques del análisis de la deserción pueden agruparse en siete categorías. Estas categorías están basadas y modificadas de varios autores:

- Psicológica: Los modelos ubicados en esta categoría hacen énfasis en las características propias de los estudiantes, en ellos se establece que la deserción es producto de un debilitamiento de las intenciones iniciales del estudiante (Monge 1981, Himmel 2002, Umaña 2004, Dubs 2005). Algunos factores personales como edad y sexo que determinan características psicológicas son ubicados por Latiesa (1992) en una categoría aparte: características personales.

- Académica personal: Se refiere a la formación previa de los estudiantes y a su record académico, en ocasiones se les considera como "competencias de los estudiantes" (Dubs 2005). Algunos autores ubican estos aspectos en características psicológicas o en características personales (Latiesa 1992).

- Sociológica: Estos enfoques enfatizan en los factores sociales externos al estudiante. El abandono se produce por la falta de integración de los estudiantes con el sistema educativo (Himmel 2002), por su origen social (Latiesa 1992).

- Económica: Aquí se analiza la relación costo-beneficio de aspectos sociales y económicos relacionados con el estar en un programa universitario. En este tipo de enfoques las universidades pueden mejorar la situación del abandono mediante programas de becas y subsidios económicos (St.John et al. 2000, Himmel 2002).

- Organizacional: El énfasis en este tipo de modelos se hace en los servicios que la universidad ofrece a los estudiantes, abarcando aspectos como acceso bibliográfico, laboratorios, calidad de la docencia, etc. (Himmel 2002).

- Oferta y demanda de la educación superior y del mercado: Se refiere a que los estudiantes pueden desertar de una institución para irse a otra a terminar sus estudios por diferentes razones de conveniencia o bien por sobreoferta de profesionales (Latiesa1992).

- Interacción: Esta categoría integra de una forma equitativa características de dos o mas categorías: tipo sicológicas, académicas personales, sociológicas, económico y organizacionales, pero en general dejan por fuera aspectos de oferta y demanda de las carreras 
universitarias y del mercado de trabajo. (Berger \& Braxton 1998, Himmel 2002, Dubs 2005).

Dentro de los análisis de deserción también hay que tomar en cuenta que el abandono está dado por procedimientos administrativos institucionales (Dubs 2005), por lo que los criterios para considerar a un estudiante como desertor varían de una universidad a otra. Además, en la literatura no siempre queda claro cual fue el criterio seleccionado para analizar la deserción, que puede referirse a la universidad, a la carrera o a un curso (Umaña 2004). Aunque no siempre se tienen claro los parámetros que se utilizaron en los estudios, se habla, a nivel de grado, de ámbitos de deserción de entre el 20 y el $60 \%$ en varios países latinoamericanos (Rivera et al. 2005, Vélez \& López 2004, Universidad de Colima 2006, Himmel 2002).

Para Costa Rica a nivel de grado se tienen datos de deserción del $65 \%$ para las 3 universidades estatales con sistema presencial en tanto que el abandono en la UNED, la única con un sistema a distancia o semipresencial, es del 80\% (Brenes 2000, Gólcher 2001, Umaña 2004). Otros datos de deserción son 50\% para la UNED de Costa Rica (Lobo 1998) y $80 \%$ para la UNED de España (García 1987). A nivel de posgrado el abandono en EEUU ronda entre el $40 \%$ y el 50\% (Lovitts \& Nelson 2000, Lovitts 2001, Dubs 2005). En el caso de posgrados a distancia en Estados Unidos se ha reportado un $44 \%$ previo a la mejora en las instrucciones de cómo estudiar a distancia y de $22 \%$ de posterior a esa mejora (Chyung et al. 1998). En Venezuela se informa de una deserción en posgrados de 93.52\% (Dubs 2005). Para Costa Rica solo se encontraron datos de la maestría académica en Extensión Agrícola (de la Universidad Estatal a Distancia) que es de carácter semipresencial y en donde se informa de una deserción del $20 \%$, y se atribuye a la falta de conocimiento de inglés de los estudiantes (Segura \& Chacón 1997).

En este trabajo se hace un análisis comparativo de la deserción por reglamento y por la intención de abandono de los estudiantes. También se analiza una serie de características de la Maestría Académica en Manejo de Recursos Naturales de la Universidad Estatal a Distancia (UNED) en relación con la deserción estudiantil para determinar en qué categoría teórica de abandono se pueden ubicar los posgrados semipresenciales.

\section{METODOLOGÍA}

Se analizó la Maestría Académica en Manejo de Recursos Naturales de la Universidad Estatal a Distancia de Costa Rica. La UNED cuenta también con un programa de bachillerato y licenciatura en Manejo de Recursos Naturales. El programa de maestría empezó a funcionar en el
2002 y al 2009 se habían inscrito 127 estudiantes repartidos en siete cohortes o promociones. Cada cohorte tenía inicialmente entre 17 y 26 estudiantes. Se trata de un programa semipresencial en el que los estudiantes deben cursar 4 bloques de materias de un cuatrimestre de duración. Cada bloque está integrado por 4 materias. Durante cada cuatrimestre se ofrecen 4 tutorías presenciales; un sábado al mes. La tutoría tiene una duración de 8 horas (2 horas por materia). La tutoría no es de asistencia obligatoria aunque generalmente la asistencia ronda entre el 95 y el $100 \%$. En aproximadamente el $75 \%$ de los cursos los estudiantes realizan, en compañía del profesor o profesora, una gira de campo que es de asistencia obligatoria y que dura uno o dos días. Por lo tanto el mayor peso del desarrollo de los cursos se hace a distancia mediante correo electrónico, teléfono y el uso de una plataforma virtual vía internet (WebCT, Microcampus o Moodle). Los estudiantes reciben una breve capacitación para el uso de las plataformas virtuales. Una vez que los estudiantes han concluido exitosamente las materias del plan de estudio, tienen 6 meses para presentar el examen de candidatura, el cual consiste en una presentación oral y escrita del diseño de su proyecto de tesis, ante un tribunal examinador. Una vez ganado el examen de candidatura los estudiantes tienen dos años para desarrollar su investigación y presentar su tesis ante el tribunal examinador. Los estudiantes que no cumplen con las fechas establecidas o que reprueban mas de un curso se consideran desertores por reglamento. Debido a la baja cantidad de graduados, no se ha aplicado el reglamento en cuanto al tiempo asignado para presentar el examen de candidatura y para defender la tesis.

Para este estudio se consideró a los 127 estudiantes que han ingresado en el programa. La información se obtuvo mediante: 1) consulta a la base de datos de la oficina de Registro de la UNED actualizada a noviembre de 2008, 2) entrevista estructurada a estudiantes, se logró entrevistar a 100 (79\% del total de la población), 3) entrevistas libres a estudiantes ( 2 entrevistas en grupo a los integrantes de la sexta cohorte, 2 entrevistas a los de la sétima y entrevista a 20 estudiantes egresados), y 4) registros internos del programa sobre la etapa en la que el estudiante deserta y tiempos transcurridos entre egresarse, presentar el examen de candidatura y defender su tesis (datos actualizados a julio de 2009).

Para evaluar cual modelo teórico sobre deserción se adapta mejor a las maestrias semipresenciales y para comprender mejor las variables que determinan el abandono se realizaron 3 tipos de análisis.

A. Se hizo un análisis del momento del programa de estudio en el que los estudiantes lo abandonan y de los tiempos que demoran entre egresarse, presentar el 
examen de candidatura y defender su tesis en relación con los tiempos estipulados por el reglamento y se comparó con la intención de abandono expresada por los estudiantes en la encuesta que se realizó. El análisis de deserción por reglamento se hizo en dos partes: primero se analizaron las primeras 5 promociones ya que son a las que según reglamento se les venció el tiempo para graduarse y después se evaluaron las cohortes posteriores para ver si se mantenía el patrón de deserción hasta que se egresaron.

B. Se evaluó una serie de variables dentro de cada una de las categorías teóricas existentes para justificar la deserción (según la intención del estudiante). Los aspectos analizados dentro de cada categoría son:

- Psicológica: edad, sexo, estado civil, intención de permanecer en la maestría, de presentar el examen de candidatura, de defender la tesis y de continuar estudios en otra universidad, razón por la que escogió esa carrera, grado de relación del trabajo con el tema de estudio.

- Académica personal: grado académico con el que ingresaron al programa y universidad en donde lo obtuvieron, nota ponderada para el ingreso, notas, cursos perdidos y aprobados, cantidad de horas de estudio, momento del día en que estudia, frecuencia de estudio, organización del tiempo de estudio.

- Sociológica: características académicas y actividades laborales del núcleo familiar, si el estudiante trabaja remuneradamente, tiempo y horario de trabajo, situaciones familiares que afecten el estudio, apoyo recibido de la entidad en donde trabaja, apoyo familiar, lugar de habitación

- Económica: ingresos económicos personales y familiares, endeudamiento, cantidad de dependientes económicos, financiamiento de los estudios

- Organizacional: calidad de la gestión administrativa, calidad de la coordinación, razón por la que escogió estudiar en la UNED, capacidad docente de los profesores, utilidad de los cursos de seminario de tesis, concordancia del perfil de salida con el mercado de trabajo, alcance del contenido de los cursos, conocimiento de los profesores, cantidad de material asignado para el estudio, grado de exigencia académica,

- Oferta y demanda de la educación superior y del mercado: cantidad de estudiantes que abandonaron estudios en la UNED para continuar en otra universidad, cantidad de estudiantes sin trabajo.

- Interacción: Presencia equitativa de aspectos sicológicos, académicos personales, sociológicos, económicos, organizacionales y de oferta y demanda de la educación superior.

C. Las variables que resultaron ser significativas para predecir la deserción en el análisis anterior fueron evaluadas de forma conjunta mediante una regresión logística binomial. Con ese fin se creó una variable dicotómica compuesta por los estudiantes que abandonan y los que permanecen en el programa según su intención expresada durante las entrevistas.

\section{RESULTADOS}

\section{A. Deserción por reglamento y tiempos empleados en la conclusión de estudios}

Considerando los plazos estipulados por reglamento, se tiene que acumulativamente: el $9 \%$ de los estudiantes desertó durante el primer bloque de materias, el 15\% abandonan antes de aprobar todos los cursos del plan de estudios, $71 \%$ no presentaron el examen de candidatura y el $87 \%$ no se graduaron $\left(\mathrm{X}^{2}, \mathrm{p}<0,01\right)$. El patrón de abandono en las últimas dos cohortes muestra que el $30 \%$ de los estudiantes abandonaron antes de concluir el programa y el $48 \%$ desertó antes de egresarse, pero hubo una mejora en la cantidad de estudiantes que presentaron examen de candidatura pues lo hizo el $43 \%$ comparado con un $29 \%$ de las promociones anteriores.

Se tiene que los estudiantes tardan 16 meses $(n=26$, min=1mes, $\max =58$ meses) en presentar el examen de candidatura después de egresarse, el tiempo estipulado por reglamento es de 6 meses, es decir que tardan casi tres veces mas de lo establecido (solo el $36 \%$ lo hizo en el plazo reglamentado). Una vez presentado y aprobado el examen de candidatura tardan 28 meses $(n=11, \min =$ 7 meses, $\max =50$ meses) en defender su tesis, lo cual no dista tanto de los 24 meses establecidos en el reglamento pero se debe considerar que la variación es muy grande.

\section{B. Análisis de las variables por categorías utilizadas para estudiar la deserción}

\section{Psicológica}

Al entrevistar a los estudiantes sobre su intención de abandonar el programa definitivamente, la deserción es de 19\% (11\% no concluyó el programa de cursos, $7 \%$ eran egresados que no presentaron el examen de candidatura y $1 \%$ presentó el examen de candidatura pero no desarrolló la tesis; $\left.X^{2}, p<0,01\right)$. De los estudiantes egresados el $86 \%$ piensa presentar el examen de candidatura, entre las principales razones por las cuales no han presentado el examen de candidatura están: se encuentran desarrollando el proyecto y aun no se les ha 
cumplido el tiempo reglamentario, no ha definido el tema, falta de tiempo, esperan respuesta de la coordinación de la maestría, se les ha pedido varias veces que reformulen su proyecto y se les presentaron problemas personales.

El 79\% considera que el programa tiene relación con el trabajo que desempeña y de ellos el $75 \%$ opina que la relación es alta. El nivel de relación manifestado por los estudiantes entre el trabajo y el estudio fue estadísticamente significativo.

Las otras variables dentro de esta categoría no fueron significativas en relación con la deserción, pero caracterizan al objeto de estudio. El 74\% indicó que el programa semipresencial le brinda la flexibilidad de horario que requiere. El 56\% de los estudiantes tiene pareja sentimental con la que conviven. Un $56 \%$ no tienen hijos y un $33 \%$ tienen uno o dos hijos. El 63\% de los estudiantes de esta maestría son hombres. La edad del $48 \%$ se ubica entre 30 y 40 años. El 55\% dice que no recibe apoyo (ni tiempo ni dinero) en el trabajo para estudiar, mientras que el 63\% afirma que no recibió apoyo de la familia. El 90\% afirmó no tener problemas familiares que les dificulte estudiar.

\section{Académica personal}

Ninguna de las variables de esta categoría determina el abandono evaluado según la intención del estudiante, pero varios de los aspectos encontrados permiten entender el proceso y generar ideas de solución. La mayor parte $(76 \%)$ de los estudiantes obtuvieron su formación universitaria en universidades públicas costarricenses. Solo el $10 \%$ de los estudiantes tenían estudios previos en la UNED y de estos solo $1 \%$ abandonó el programa por considerar el nivel muy bajo, pero la cantidad de casos no es suficiente para ser concluyente.

Los grados académicos con los que iniciaron el programa corresponde a: $56 \%$ licenciatura, 35\% bachillerato y $9 \%$ maestría. En el caso de los que tenían una maestría al iniciar, desertaron el $44 \%$, en tanto que los que poseían bachillerato o licenciatura la deserción fue de 14\% y 16\% respectivamente. Al agrupar los estudiantes que iniciaron la maestría con un grado académico y compararlos con los que iniciaron el programa teniendo un posgrado se encontró una diferencia significativa en cuanto a su permanencia $\left(X^{2}, p=0,03\right)$, no obstante, los datos deben analizarse con precaución por la baja cantidad de casos.

Los estudiantes del programa estudian en promedio 19,1 horas a la semana $(D E=9,1, \min =5, \max =40)$. El $91 \%$ estudia entre semana, en las noches (53,7\%). Para la organización del estudio el $73 \%$ prepara un cronograma pero solo suelen concretarlo el $47 \%$.

El promedio de notas de los estudiantes es de 8,8 (DE=0,6, $\min =5, \max =9.7)$. Solo hay un $3,5 \%$ de materias perdidas $y$ un
4,5\% de materias en donde el estudiante no se presentó. Hay dos grupos en los que se concentra la mayor cantidad de materias reprobadas: 1 ) el primer bloque de materias (40\%) y 2) los tres seminarios de tesis (25\%).

\section{Sociológica}

Ninguna de las variables de esta categoría determina el abandono evaluado según la intención del estudiante, pero describen a la población estudiada. La mayoría de los estudiantes $(87 \%)$ viven en las provincias centrales (San José, Heredia, Alajuela y Cartago). En el núcleo familiar de los estudiantes existe un alto grado académico: 91\% de las parejas sentimentales con las que conviven, $42 \%$ de las madres y el $78 \%$ de los padres poseen estudios universitarios.

El 95\% de los estudiantes trabaja. Solo se presentó deserción entre las personas que trabajan remuneradamente. El 84\% trabaja tiempo completo. El 45\% trabaja en instituciones públicas. La mayoría trabaja una jornada de lunes a viernes (84\%). El 88\% de las parejas de los estudiantes trabaja, el $61 \%$ lo hace en el campo profesional, científico e intelectual. Las madres de los estudiantes son amas de casa (50\%) o están el campo profesional, científico e intelectual (27\%). Los padres tienen actividades mas variadas: en el campo científico e intelectual (33\%), en el nivel técnico o profesional medio (16\%), venta de locales y prestación de servicios directos (16\%).

\section{Económica}

No se detectaron problemas de abandono relacionados con aspectos económicos personales y familiares. El $64 \%$ de los estudiantes cuenta con casa, $36 \%$ paga préstamo de vivienda. En el 69\% los estudiantes tienen ingresos (del núcleo familiar) entre quinientos mil y un millón y medio de colones, (el salario base para un bachiller universitario en julio de 2009 es de 361.664 colones; Gobierno de Costa Rica 2009). En $81 \%$ de los casos el estudiante y su pareja son el principal sostén económico familiar. El 91\% de los estudiantes se financia los estudios por su propia cuenta; el $85 \%$ mediante recursos propios y el $8 \%$ mediante préstamo.

\section{Organizacional}

En esta categoría hubo dos variables determinantes en el abandono. La primera fue la capacidad docente de los profesores. Únicamente el $42 \%$ de los estudiantes estuvo de acuerdo en que los profesores tienen capacidad para enseñar. La segunda fue el grado de concordancia entre el perfil de salida profesional y el mercado laboral. Apenas un $29 \%$ estuvo de acuerdo en que el perfil de salida es acorde con el mercado de trabajo. Además, se 
debe considerar que de los estudiantes que abandonaron el programa de forma definitiva lo hicieron debido a que consideraron que el nivel era muy bajo (61\%) o porque se desmotivó (22\%) por tanto cambio que le pidieron en su proyecto de investigación.

Los siguientes elementos no fueron significativos para determinar el abandono. La gestión administrativa del programa fue considerada como buena o muy buena por el 70\% de los entrevistados y la coordinación de la carrera por el $60 \%$. El nivel de conocimiento de los profesores fue calificado como muy bueno por el $53 \%$ de los estudiantes. No obstante, el $72 \%$ de los estudiantes cursaron las materias de seminario de tesis I, II y III, de ellos, el $74 \%$ califican entre nada, poco y regular el aporte de estos cursos para el desarrollo de su proyecto de graduación. El grado de exigencia académica fue alta para un $48 \%$ y solo el $46 \%$ estuvo de acuerdo en que los contenidos de los cursos cubren de forma completa el objeto de estudio de la Maestría, pero el $35 \%$ opinó que la cantidad de contenidos asignados por materia durante el cuatrimestre era excesiva.

\section{Oferta y demanda de la educación superior y del mercado}

El $6 \%$ de los estudiantes abandonó la maestría de la UNED para continuar sus estudios en otra universidad. Las razones que dieron son: le pareció que daban lo mismo a nivel de grado, la calidad de la UNED le pareció muy mala, encontró una universidad con mejor nivel, le ofrecieron una maestría más interesante, quería cambiar a una modalidad presencial y quiere asistir a otra universidad. Los destinos escogidos por estos estudiantes para continuar los estudios son: la Universidad Autónoma de Monterrey (México), la Universidad de Costa Rica (Costa Rica), la Universidad Complutense de Madrid (España) y la Universidad Interamericana (Costa Rica).

\section{Interacción}

Se encontró que la intención de abandono del programa se determina por tres variables (ver siguiente apartado). Dos pertenecen a la categoría organizacional (capacidad docente de los profesores y grado de concordancia entre el perfil de salida profesional y el mercado laboral) y otra a la psicológica (grado de relación entre el trabajo y el programa).

Aunque se cumple el requisito de tener variables de dos o mas categorías, el aporte de cada una no es equitativo según el modelo estadístico desarrollado.

\section{Regresión logística binomial}

Nueve variables mostraron relación con la deserción. Tres de ellas tenían pocos casos por lo que no fueron in- cluidas en la regresión logística binomial. Estas fueron: 1) no tener trabajo remunerado, que se asoció con la permanencia en el programa, 2) ingresar al programa teniendo el grado de maestría, que se asoció con el abandono y 3) tener estudios previos en la UNED, que se asoció con la permanencia. Las variables evaluadas son:

- Nivel de relación entre el trabajo que desempeña y el programa

- Grado de concordancia entre el perfil de salida profesional y el mercado laboral

- Grado en que el contenido de los cursos cubren el objeto de estudio

- Grado de exigencia académica

- Nivel de conocimiento de los profesores

- Capacidad docente de los profesores

La ecuación resultante del análisis es:

$P=1 /\left(1+e^{\left(-3.71+1,875^{*} \text { Variable1+3,329*Variable } 2,264^{*} \text { Variable } 3\right)}\right)$

Donde $\mathrm{P}$ es la probabilidad de abandono, si $\mathrm{P}$ es igual o mayor a 0,5 se considera que existe riesgo de que el estudiante abandone.

Las variables que estadísticamente determinan la deserción fueron:

- Grado de concordancia entre el perfil de salida profesional y el mercado laboral $(p=0,009)$ (Variable 1$)$

- Capacidad docente de los profesores $(p=0,001)(\mathrm{Va}-$ riable 2)

- Nivel de relación entre el trabajo que desempeña y la maestría $(p=0,003)$ (Variable 3$)$

Estas variables mostraron una correlación negativa, lo que indica que entre mas negativa sea la opinión de los estudiantes en estos aspectos, mas probable es que abandonen el estudio. El modelo estadístico no es muy sensible $\left(r^{2}\right.$ de Negelkerke $\left.=0.435\right)$ esto indica que no se logró identificar todas las variables que contribuyen a la deserción. No obstante, el modelo tiene un poder de clasificación alto (Poder de clasificación $(0,95)=0,88$ ).

\section{DISCUSIÓN}

\section{¿Retraso o Deserción?}

Con frecuencia el porcentaje de deserción es calculado de acuerdo con los lineamientos establecidos en el reglamento académico (Dubs 2005). Los datos obtenidos de esa manera enmascaran problemas asociados con el re- 
traso en la graduación y traslado a otras instituciones (Latiesa 1996). De esta forma se generan cifras muy variables y que se refieren a la deserción de una forma muy amplia. En el caso de esta maestría, se encontró que es más importante la prolongación de los estudios que el abandono (deserción por reglamento 87\%, intención de abandono $19 \%$-del cual $6 \%$ corresponde a traslado a otras instituciones-, prolongación de estudios 68\%). No obstante, todos los datos obtenidos se encuentran dentro de los ámbitos reportados en la literatura: deserción 8 a 93.52\% (Latiesa 1996, Dubs 2005), retraso 1 a 100\% (Latiesa 1996, Cabrera et al. 2006) y traslado a otras instituciones 4.2\% (Cabrera et al. 2006). Basados en su experiencia y en la ejecución de algunas estrategias algunos investigadores calculan que el porcentaje de deserción en posgrados universitarios presenciales y semipresenciales puede disminuir a un 22 o 25\% (Lovitts \& Nelson 2000, Lovitts 2001, Chyung et al. 1998), sin embargo, no consideraron el traslado a otras instituciones. Por lo tanto un análisis cuidadoso, la ejecución de estrategias adecuadas y dando el tiempo necesario para que estas tengan efecto, el porcentaje de deserción sensus lato puede bajar a un 15\% (deserción por intención menos deserción por traslado a otras universidades).

\section{Deserción sensu stricto}

\section{Relación con la teoría}

En general los autores estadounidenses han analizado la deserción considerando las categorías y/o modelos teóricos (St. John 2000, Reason 2003), dándole, énfasis a la interacción social (Metzner \& Bean 1987, Berger \& Braxton 1998, Lovitts \& Nelson 2000, Lovitts 2001). No obstante, los estudios realizados en América Latina, al igual que en este, integran una serie de variables pertenecientes a diferentes categorías teóricas y se ha encontrado que las que determinan la deserción varían para cada país y para cada institución (Himmel 2002, Dubs 2005, Rivera Rearte et al. 2005, Cabrera et al. 2006). Para el estudio de la deserción en universidades latinoamericanas se deben analizar las variables en una forma holística, dentro del contexto sociocultural e institucional de cada caso, y se debe buscar la solución de los problemas específicos detectados.

\section{Medidas propuestas}

En este caso, se identificaron tres variables asociadas con el abandono por intención del estudiante y se proponen las siguientes medidas: 1) mejorar la capacidad de los docentes para hacerse entender en un sistema semipresencial; 2) revisar el perfil de salida de la carrera lo cual implica mejoras en todo el diseño curricular; y 3) mejorar el sistema de selección de los estudiantes que entran al programa. Respecto a este último punto, es importante notar que debe hacerse después de corregir los errores del sistema pues no es justo atribuirle al estudiante los problemas organizacionales (Donoso \& Schiefelbein 2007), además ya se ha demostrado que hacerlo a la inversa no mejora las tasas de deserción (Lovitts \& Nelson 2000).

A partir del análisis de regresión, la varianza explicada fue de $43.5 \%$ por lo que no se logró identificar todas las variables asociadas con la deserción, pero según el análisis cualitativo hay otras variables que deben considerarse en la solución y/o en futuras investigaciones: 1) revisar si existe una sobre carga académica y/o laboral y si está asociada a la deserción (Himmel 2002); 2) analizar el contenido y estructura del primer bloque de materias (Chyung et al. 1998, Ishitani 2003); 3) promover el ingreso de estudiantes de grado del mismo programa (Chyung et al.1998, Park 2005) y 4) mejorar la inducción de estudiantes a un sistema semipresencial (Chyung et al.1998).

\section{Retraso en la graduación}

El atraso en la graduación está relacionado con los problemas que los estudiantes tienen después del primer bloque de materias y con el desarrollo de los proyectos de investigación (Lovitts \& Nelson 2000, Lovitts 2001). Por lo tanto, las medidas que pueden aminorar esta problemática son: 1) reestructurar los tres cursos de seminarios de tesis; 2) fortalecer el desarrollo de investigaciones en todas las asignaturas; 3) mejorar el acompañamiento que se les da a los estudiantes durante todo el desarrollo de su proyecto de investigación (Lovitts \& Nelson 2000, Lovitts 2001); 4) ajustar los tiempos reglamentarios ya que no se adaptan a la realidad de una maestría académica en la que los estudiantes trabajan tiempo completo (Himmel 2002); y 5) favorecer la selección de estudiantes que realmente desean realizar una maestría académica y no una profesional.

\section{Continuidad de estudiantes del programa de grado y maestría}

Por otra parte, es interesante notar que en comparación con los estudiantes de grado de la UNED los de posgrado tienen familias con perfiles diferentes tanto económico como académico y laboral (Umaña 2004) lo que puede ser reflejo de una dualidad en la misión y visión efectiva dentro de la misma institución. Hasta la fecha solo el 10\% de los estudiantes tienen estudios previos en la UNED. Si en algún momento se mejora la situación mediante el fortalecimiento del sistema de becas, debe considerarse que en EEUU se ha encontrado que los estudiantes que permanecen son los que, como parte del acuerdo de beca, colaboran en docencia e investigación (Lovitts \& Nelson 2000, Lovitts 2001). 


\section{Conclusiones}

Analizar las razones que motivan a un estudiante a desertar de su programa de estudios es una tarea complicada pues concluimos que muchas veces se confunde la deserción con el retraso en la graduación. Los problemas de deserción y prolongación de estudios deben analizarse por separado. Además, debe considerarse que los estudiantes de posgrados semipresenciales que laboran tiempo completo presentan condiciones diferentes a los de posgrados presenciales que no laboran, por lo que los diseños curriculares y los tiempos reglamentarios deben responder a esa realidad.

\section{AGRADECIMIENTOS}

Se agradece el trabajo asistencial a Maribel Zúñiga Solís, Alexandra Gólcher Vargas, Marisela Calderón Pérez y Jenifer Salazar Cruz. Daniela Castro Cabalceta colaboró con la recolección, procesamiento y sistematización de la información. Ligia Jeannette Bermúdez Mesén y Benicio Gutiérrez Doña colaboraron dando asesoría para realizar el análisis estadístico. Víctor Méndez Estrada y un revisor anónimo hicieron valiosos comentarios para mejorar el documento.

\section{REFERENCIAS}

Allen, D. 1999. Desire to finish college: an empirical link between motivation and persistence. Research in higher education 40: 461-485. (También disponible en línea: http://web.ebscohost.com/ehost/pdf?vid=2\&hid=8\&s $\mathrm{id}=7 \mathrm{e} 574 \mathrm{be} 5-5 \mathrm{f} 8 \mathrm{a}-434 \mathrm{f}-8 \mathrm{c} 73-5140 \mathrm{~d} 088 \mathrm{~b} 68 \mathrm{e} \% 40$ sess ionmgr10)

Bean, J. 1980. Dropouts and turnover: The synthesis and test of a causal model of student attrition. Research in higher education 12: 155-187. (También disponible en línea: http://www.eric.ed.gov/ERICDocs/data/ericdocs2sql/content_storage_01/0000019b/80/31/9b/fc.pdf)

Berger, J. \& J Braxton. 1998. Revising Tinto's interactionalist theory of student departure through theory elaboration: Examining the role of organizational attributes in persistence process. Research in Higher education 39: 103- 119. (También disponible en línea: http://web. ebscohost.com/ehost/pdf?vid $=5 \&$ hid $=8 \&$ sid $=$ fd 8873 ac-6b08-4d51-91b8-05c6cd38a9ed\%40sessionmgr10

Brenes, I. 2000. Aspectos relacionados con el rendimiento académico de los estudiantes que ingresaron a las instituciones de educación superior universitaria estatal en 1990. San José: Consejo Nacional de Rectores, Oficina de Planificación.

Cabrera, L., J.T. Bethencourt, M. González \& P. Álvarez. 2006. Un estudio transversal retrospectivo sobre prolongación y abandono de estudios universitarios. RELIEVE 12:105-
127. (También disponible en línea: http://www.uv.es/ RELIEVE/v12n1/RELIEVEv12n1_1.htm

Chyung, Y., D. Winiecki \& A. Fenner. 1998. A case study: Increase enrollment by reducing dropout rates in adult distance education. In Distal learning '98. Proceedings of the annual conference in distance teaching and learning (14th Madison, WI, August 5-7, 1998). (También disponible en línea: http://www.eric.ed.gov/ERICDocs/data/ericdocs2sql/content_storage_01/0000019b/80/15/bf/a9.pdf)

Donoso, S. \& E. Schiefelbein. 2007. Análisis de los modelos explicativos de retención de estudiantes en la universidad: una visión desde la desigualdad social. Estudios pedagógicos 23: 7-27. (También disponible en línea: http://mingaonline.uach.cl/pdf/estped/v33n1/art01.pdf)

Dubs, R. 2005. Permanecer o desertar en los estudios de postgrado: Síntesis de modelos teóricos. Investigación y postgrado 20: 55-79. (También disponible en línea: http:// redalyc.uaemex.mx/src/inicio/ArtPdfRed.jsp?iCve $=65820$ 103\&iCveNum=3533)

García, L. 1987. Rendimiento académico y abandono en la educación superior a distancia. Estudios de Educación a Distancia No. 10. Universidad Nacional de Educación a Distancia, Madrid, España.

Gobierno de Costa Rica. 2009. Decreto 35370 MTSS. La Gaceta 136, 15 de julio de 2009. (También disponible en linea: http://www.construccion.co.cr/dirtecnica/Salarios_Minimos_2do_semest_2009.pdf)

Gólcher, R. 2001. Elevada deserción universitaria. La Nación, San José, Costa Rica, Jun 21. (También disponible en línea: http://www.nacion.com/In_ee/2001/junio/17/pais2.html

Himmel, E. 2002. Modelos de análisis para la deserción estudiantil en la educación superior. Calidad de la Educación: Retención y movilidad estudiantil en la educación superior. $2^{\circ}$ semestre. 91-107. (Tambien disponible en linea: http://www.cse.cl/public/Secciones/seccionpublicaciones/doc/35/cse_articulo141.pdf)

Ishitani, T. 2003. A longitudinal approach to assessing attrition behavior among first-generation students: time varying effects of pre-collage characteristics. Research in higher education 44: 433-449. (También disponible en línea: http://web.ebscohost.com/ehost/pdf?vid=5\&hid=8\&sid $=$ bc28412f-4d2c-47c9-a564-463e533f3b55\%40sessionm gr11)

Latiesa, M. 1992. La deserción universitaria. Desarrollo de la escolaridad en la enseñanza superior. Éxitos y fracasos. Editorial Siglo XXI. España. (También disponible en línea: http://www.cis.es/cis/opencm/ES/3_publicaciones/catalogo/ver.jsp?id=64

Latiesa, M. 1996. Tipología y causas de la deserción universitaria y el retraso en los estudios. Revista Diálogo Iberoamericano, 5. (También disponible en línea: http://dialogo.ugr. es/anteriores/dial05/11-5.htm.)

Lobo, N. 1998. El problema de la retención escolar en el sistema universitario a distancia costarricense: el caso de la UNED. Tesis Doctoral en Educación, Universidad Nacional 
de Educación a Distancia, Madrid, España.

Lovitts, B. \& C. Nelson. 2000. The Hidden Crisis in Graduate Education: Attrition from Ph.D. Programs, Academe 6:44- 50. (Tambien disponible en linea: http://www.aaup.org/ AAUP/pubsres/academe/2000/ND/Feat/lovi.htm?PF=1

Lovitts, B. 2001. Leaving the Ivory tower: The causes and consequences of departure from doctoral study. Rowman \& Littlefield. Boston. EE. UU. (También disponible en línea: http://findarticles.com/p/articles/mi_qa3752/is_200205/ ai_n9052565/pg_2/?tag=content;col1)

Lovitts, B. 2005. How to grade a dissertation. Academe 6:18-23. (También disponible en línea: http://www.aaup.org/ AAUP/pubsres/academe/2005/ND/Feat/lovi.htm

Méndez, V. 1997. Deserción estudiantil en los Programas de Extensión Abierta de la Dirección de Extensión de la UNED: 19881993. Rev. Innovaciones Educativas UNED 8: 71-80.

Metzner, B. \& J. Bean. 1987. The estimation of a conceptual model of nontraditional undergraduate student attrition. Research in Higher Education 27: 15-38. (También disponible en línea: http://www.springerlink.com/content/ r24xv4l27784p766/)

Monge, A. 1981. Algunos factores causantes de deserción estudiantil en los cuatro primeros meses del primer ano de estudios en los anos de 1973 a 1978, en el Instituto Tecnológico de Costa Rica. Tesis de licenciatura en ciencias de la educación, con énfasis en orientación. Facultad de Educación. Escuela de Orientación y Educación Especial. Universidad de Costa Rica.

Montero, E., J. Villalobos \& A. Valverde. 2007. Factores institucionales, pedagógicos, psicosociales y sociodemográficos asociados al rendimiento académico en la Universidad de Costa Rica: un análisis multinivel. Revista electrónica de investigación y evaluación educativa RELIEVE 13: 215 234. (También disponible en línea: http://www.uv.es/RELIEVE/v13n2/RELIEVEv13n2_5.htm)

Murtaugh, P., L. Burns \& J. Schuster. 1999. Predicting the retention of university students. Research in higher education 40: 355-371. (Tambien disponible en linea: http://web. ebscohost.com/ehost/pdf?vid=5\&hid=5\&sid=fe9ab2a996b7-4463-90da-c8cc41fdf631\%40sessionmgr11)

Park, C. 2005. War of attrition: patterns of non-competition amongst postgraduate research student. Higher Education Review, 38: 48-53. (Tambien disponible en linea: http:// search.ebscohost.com/login.aspx?direct=true $\& \mathrm{db}=$ ehh $\&$ $\mathrm{AN}=19070044$ \&amp;lang=es\&site=ehost-live)

Reason, R. 2003. Student variables that predict retention: recent research and new developments. NASPA Journal 40: 172191.(También disponible en línea: http://web.uccs.edu/ bgaddis/retention/Student\%20Variables\%20that\%20Pre dict\%20Retention.pdf)

Rivera, E., H. Roca, B. Echart, A. López, S. Sossa, C. Mercado \& B. Barrera. 2005. Estudio sobre repitencia y deserción en la educación superior en Bolivia. UNESCO. (También disponible en línea: http://unesdoc.unesco.org/images/0013/ 001397/139799s.pdf)

Segura, M. \& M. Chacón. 1997. Resultados de la Evaluación del Programa de Maestría en Extensión Agrícola. EUNED. San José, Costa Rica.

St. John, E., A. Cabrera, A. Nora, \& E. Asker. 2000. Economic Influences on Persistence Reconsidered. How can finance research inform the reconceptualization of persistence models? In J.M. Braxton (Ed) Reworking the student departure puzzle. Nashville: Vanderbilt University (Tambien disponible en linea: http://www.education.umd.edu/ EDHI/about/faculty_pages/cabrera/Economic\%20Influe nces\%20in\%20Persistence.pdf

Umaña, R. 2004. Elaboración de una propuesta contextual, teórica y metodológica para el abordaje de la deserción de estudiantes en la Universidad Estatal a Distancia de Costa Rica. Tesis para optar por el grado de Licenciatura, Facultad de Ciencias Sociales, Escuela de Antropología y Sociología, Universidad de Costa Rica.

UNESCO. 2009. 2009 World Conference on Higher Education: The New Dynamics of Higher Education and Research For Societal Change and Development (UNESCO, Paris, 5 - 8 July 2009). Communique (8 July 2009). (Tambien disponible en linea: http://www.unesco.org/fileadmin/ MULTIMEDIA/HQ/ED/ED/pdf/WCHE_2009/FINAL\%20CO MMUNIQUE\%20WCHE\%202009.pdf)

Universidad de Colima. 2006. La deserción en la educación superior. Observatorio 27 noviembre 2006. (También disponible en línea: http://www.ucol.mx/observatorio/comunicados/Comunicado7.pdf)

Vélez, A. \& D. López. 2004. Estrategias para vencer la deserción Universitaria. Educación y Educadores. 7: 177-204. (También disponible en línea: http://educacionyeducadores. unisabana.edu.co/index.php/eye/article/view/316/419 
Research Journal of the Costa Rican Distance Education University (Online Edition ISSN: 1659-441X) Vol. 1(2), December, 2009 